\title{
The Heart of an Image: Quantum Superposition and Entanglement in Visual Perception
}

\author{
Jonito Aerts Arguëlles \\ KASK and Conservatory, \\ Jozef Kluyskensstraat 2, 9000 Ghent, Belgium \\ E-Mail: jonitoarguelles@gmail.com
}

\begin{abstract}
We analyse the way in which the principle that 'the whole is greater than the sum of its parts' manifests itself with phenomena of visual perception. For this investigation we use insights and techniques coming from quantum cognition, and more specifically we are inspired by the correspondence of this principle with the phenomenon of the conjunction effect in human cognition. We identify entities of meaning within artefacts of visual perception and rely on how such entities are modelled for corpuses of texts such as the webpages of the World-Wide Web for our study of how they appear in phenomena of visual perception. We identify concretely the conjunction effect in visual artefacts and analyse its structure in the example of a photograph. We also analyse quantum entanglement between different aspects of meaning in artefacts of visual perception. We confirm its presence by showing that well elected experiments on images retrieved accordingly by Google Images give rise to probabilities and expectation values violating the Clauser Horne Shimony Holt version of Bell's inequalities. We point out how this approach can lead to a mathematical description of the meaning content of a visual artefact such as a photograph.
\end{abstract}

Keywords: Quantum theory; Quantum cognition; Visual perception; Conjunction effect; Quantum entanglement; Bell's inequalities.

\section{Introduction}

I have a long-standing interest in ephemeral and emergent phenomena of visual perception. From how the zoetrope produces the movement out of still images and hence led to the birth of cinema to the way in which holography makes emerge a three-dimensional view from a laser and a two-dimensional photographic plate. The end-work for my Master study in visual art was focused on the phenomenon of the afterimage [1]. This phenomenon takes place on the interface of the interaction between observer, the one who looks, and object, that which is looked at, in a way so that they produce 'emergences' - the zoetrope leads in an unexpected way from still images to 'movement', holography gives rise to a third dimension from a picture that is two-dimensional, afterimages both positive and negative are self-emergent. I can date back my fascination for these emergent phenomena of visual perception to my original passion for visual art in general and photography specifically. Indeed, even with the simple action of a photograph, it is always the elusive nature of that moment that continues to intrigue me. A photograph is a snapshot of reality, but it is at the same time something much deeper and more meaningful than that moment in itself. As photographer, one never sees the exact image that is recorded. The moment one presses the shutter, the mirror folds up and one sees no longer anything through the camera. 
When photography was introduced one hundred and fifty years ago, there was a tendency to consider it as a perfect medium to document reality. This was because the mechanical quality of the process ensured an unspoiled replica of the subject. The technical progress of the following decades and the rise of photojournalism made for an even clearer and more real looking product. But of course, it is not a duplicate of reality at all. Photographers are now able to edit their photographs digitally, although we should not forget that in the time of analogue film editing was already frequent, albeit less sophisticated. At that time, for example, there were various ways of developing film and adjustments could be made when printing images. In this way, an image can always be interpreted as the 'look of the photographer'. He chooses at least partly what we see, and also what gets the most attention in the image. The image therefore carries more in it than just the literal representation of reality. There is more going on when looking at an image than pure registration. It is this elusive 'more' that fascinated me so much already as a boy when I was making my first pictures.

This fascination has recently been triggered to a new level by an experience of scientific collaboration within the framework of the Center Leo Apostel of the Free University of Brussels with a group of mainly physicists, but also deeply involved in interdisciplinary applications of the natural sciences. Not so long ago, one of these physicists published an article entitled 'Ephemeral properties and the illusion of microscopic particles' 2]. The article shows how some of the properties of quantum particles can be considered to be ephemeral. The history of quantum mechanics is indeed intertwined with a search for the peculiar nature of quantum entities and their properties [3, 4, 5, 6]. There is a consensus among the physics' experts on the 'elusiveness of this peculiarity', and more generally 'the incomprehensibility of the behaviour of quantum entities themselves'. One among the famous ones, Richard Feynman, once expressed such 'incomprehensibility and vagueness of the mysterious quantum world' as follows: "I think I can safely say that nobody understands quantum mechanics."

It would lead us too far to even give an overview of this situation of the ephemeral and the elusive in the broad field of quantum mechanics. However, the collaboration in which I became involved was focused on a specific sub-research field that is called 'quantum cognition'. Strangely enough, this sub-study sheds light on a specific aspect of the quantum nature, directly linked to the collection of phenomena of human visual perception in which I was involved. I mentioned already the notion of emergence and the first quantum effect I would like to consider is related to this phenomenon.

\section{The non-summability of visual perception}

Let me first clarify what the title of this section refers to. It specifies the general truth that "the whole is greater than the sum of its parts' (hence the term "non-summability"), except in the domain where classical set theory applies. Indeed, if we consider two sets

$$
A=\{\text { Cat }, \text { Horse, Donkey }\} \quad B=\{\text { Cat, Mouse, Squirrel }\}
$$

then these two sets form a whole that in mathematics is called 'their union'

$$
A \cup B=\{\text { Cat, Horse, Donkey, Mouse, Squirrel }\}
$$

which is simply the sum of the two original sets as parts of such whole. One could argue that the two parts contain each three elements while the union contains only five elements, indeed, Cat is present in both parts, whereas it only appears once as an element of their union. In this sense, the use of the word 'sum' is slightly misleading, which is why 'union' has been introduced as a concept in set theory. The expression that 'the whole is greater than the sum (or union) of its parts' means that 'by the coming together of the parts, something new is created that makes an essential contribution to the whole'. 
Let me also express the dual aspect of 'the whole is greater than the sum of its parts', because the examples we will later deal with mainly refer to it. For the sets $A$ and $B$ we can see that the intersection

$$
A \cap B=\{C a t\}
$$

is always smaller than each of the $A$ and $B$ collections separately. This set-theoretical law that 'the intersection is always lesser than each of its elements' can also lead to problems in those domains where classical set theory does not apply, where an intersection can behave as if it was larger than one of its elements, or the other. As I said, this is the dual version of the phenomenon where the whole appears to be larger than the sum of its parts. In the course of the last century, it became clear during a continuous in-depth investigation into the structure of quantum mechanics that the latter carries inherently in its

principles this 'whole being greater than the sum of its parts' aspect. More specifically, if two entities $A$ and $B$ are brought together, then the new composite entity $C$, as the whole formed from parts $A$ and $B$, is different (larger) than their sum (union), so we can write

$$
C>A \cup B
$$

It is a well-defined principle in quantum mechanics, called 'the superposition principle', which is at the origin of this phenomenon of the whole being greater than the sum of its parts. A clear analysis of this 'quantum phenomenon of magnification of the whole after composition of its parts', which goes into all the necessary details, has only been recently established. However, it was already taken into due account in the early years of quantum mechanics, although rather at an intuitive level, as evidenced by the book written by Werner Heisenberg, one of the founders of quantum mechanics, with the title: "The part and the whole" [3. In quantum cognition, and therefore also in the research in which I participated at the Center Leo Apostel, effective use is being made of this specific quantum feature, to build models from the mathematics of quantum mechanics for still unknown phenomena of human cognition. Let me look at some of these phenomena, so that I can use them to make it clear what the possible applications are for the field of visual perception.

\section{The Linda problem}

The first example I will consider is in the field of human cognition, and it has already been extensively dealt with in quantum cognition approaches. In the 1990s, psychologists Amos Tversky and Daniel Kahneman discovered and identified the cognitive phenomenon called the 'conjunction fallacy' [7]. A well-known example of the conjunction fallacy, which has meanwhile been considered archetypical, is called the 'Linda problem'. It is about asking the following:

"Linda is 31 years old, single, outspoken, and very bright. She majored in philosophy. As a student, she was deeply concerned with issues of discrimination and social justice, and also participated in anti-nuclear demonstrations. Which is more probable? $(A)$ Linda is a bank teller. $(B)$ Linda is a bank teller and is active in the feminist movement."

The majority of individuals subjected to the above 'Linda problem' alternative opt for option $(B)$. However, the probability that two events occur together, i.e. in 'conjunction', is always less than or equal to the probability that one of the two occur alone. Indeed, for the two events $(A)$ and $(B)$, this can be written as the two inequalities:

$$
P(A \text { and } B) \leq P(A) \quad P(A \text { and } B) \leq P(B)
$$


whereas people who are presented with the Linda problem will generally claim that

$$
P(A \text { and } B)>P(A)
$$

Let us take a brief look at some concrete values for these probabilities, to see what 'error' is being made. Suppose there is only a very small probability that Linda is a bank teller, for example $P(A)=0.05$ and a high probability that Linda is feminist, for example $P(B)=0.95$, then the probability that Linda is both must anyhow be smaller than each of the two separately, and if they are independent of one another, this probability must be equal to the product $0.05 \times 0.95=0.0475$, and this is smaller than 0.95 and smaller than 0.05. What happens if an overwhelming majority of those questioned assesses these probabilities in such a wrong way? The classical answer is that the interviewees make a 'probability judgment error', hence the name 'conjunction fallacy' given by Tversky and Kahneman to the phenomenon. To measure the importance of the phenomenon, we note that the 'conjunction fallacy' occurs very often in stories like the one about Linda, and is therefore not at all the result of a deliberate 'deception' of the interviewees [8]. In quantum cognition, a totally different explanation is put forward. It is not assumed that the interviewees make a mistake in their assessment, on the contrary, they are considered to be 'correctly judging' what the probabilities in question are.

In order to be able to clarify the basis of this totally different and new way of considering this phenomenon of 'conjunction fallacy', from the perspective of quantum cognition, it will be useful to first consider a second example, which I myself have been involved with when working with the group of researchers at the Center Leo Apostel of the VUB. This second example, situated in the field of concept theory research in psychology, shows on a deeper level what is happening in the formation of a "conjunction that seems greater than one of its elements', as is the case for the Linda problem. After all, there too, a decade before the conjunction fallacy was identified by Tversky and Kahneman, a peculiarity was noted in connection with the conjunction of two concepts, which was called 'the pet-fish problem'.

\section{The pet-fish problem}

In 1981, Daniel Osherson and Edward Smith studied the combination of the two concepts Pet and Fish in the conjunction Pet-Fish and considered 'how typical' different examples of the concepts of Pet, Fish and Pet-Fish are estimated [9]. They noticed that examples such as Guppy and Goldfish are much more typical of the Pet-Fish conjunction than is the case for the separate concepts of Pet and Fish, and showed that this is in contradiction with a modelling of the situation by classical fuzzy set theory. Indeed, as is the case for the probabilities in the Linda problem, fuzzy set theory also demands that the typicality of an example with respect to the conjunction of two concepts is less or equal to the typicality of that example for each of the concepts taken separately. And since Guppy was the item that Osherson and Smith put forward with respect to the pet-fish problem, the phenomenon is also often called the 'guppy effect'.

What is important, and in the early stages of the investigation was not clear, is that this guppy effect is very often present in combinations of two concepts. For example, studies have shown that respondents believe that Olive is a more typical example of Fruits and Vegetables than of Fruits, or Vegetables, considered separately (see Table 4a of [10]), and this is also the case for Mint with respect to Food and Plant and Tree House with respect to Building and Dwelling (see [11] and Table 4 of [12]). Not so however for Apple, which is found to be more typical of Fruits than of Fruits and Vegetables, or of Vegetables, while respondents find Broccoli to be a more typical example of Vegetables than of Fruits and Vegetables, or of Fruits. But even Apple is found to be more typical of Fruits and Vegetables than of Vegetables and Broccoli is found to be more typical of Fruits and Vegetables than of Fruits (see Table 4a of [10]). Psychologists who study concepts and their conjunctions [11, called 'overextension' the effect where an exemplar (such as Apple) is more typical for the conjunction of two concepts (such as Fruits and Vegetables) as for one of the concepts 
apart (such as Vegetables), and found it to be a very frequent phenomenon (see Tables 1a, 2a, 3a and 4a of [10] and Table 4 of [12]), so certainly not a rare phenomenon as Osherson and Smith could still think back in 1981. The exemplars Olive, Mint and Tree House are 'double overextended' respectively with respect to the concepts combinations Fruits and Vegetables, Food and Plant and Building and Dwelling, hence they are found to be more typical with respect to the conjunction as with respect to both concepts aparts. Such a double overextension, although it occurs systematically, is not abundant like it is the case for a single overextension. By analyzing the examples of overextension, and more even so for the examples of double overextension, in detail, we can understand much better what is going on and why quantum cognition is able to bring forward a fundamental solution for the 'conjunction phenomenon in general', namely that 'the conjunction can be perceived to be larger than its composing elements'.

In order to understand what is going on, let us consider the conjunction Fruits and Vegetables. Examples that show overextension, and certainly so the ones that show double overextension, contain a typicality of what the conjunction introduces at the level of meaning itself, let us illustrate this by means of the example Olive. For an olive, we can say that there are doubts whether it is a fruit or a vegetable, and therefore, because there is such doubt, an olive is more typical of Fruits and Vegetables than of Fruits, or of Vegetables. It is as if Olive was located in an area where Fruits and Vegetables introduce something that is 'new', also from a structural viewpoint, which is neither in the area of Fruits nor in the area of Vegetables, so not in what we call the traditional conjunction. This 'new' aspect manifests as a consequence of 'the specific meaning of the conjunction' which allows to bring doubtful cases into its realm, while the meaning of the conjunction based on set theory and hence logic 'does not allow such doubtful cases'. From the viewpoint of the latter, something can be part of it only if it is in 'both', i.e. part of Fruits and at the same time part of Vegetables, and not if it is a situation of doubt, i.e. perhaps part of Fruits or perhaps part of Vegetables. That is precisely what is allowed in quantum cognition. This is also the case for Mint and Tree House, they give rise to such a doubt, respectively with respect to being a plant or food, and being a building or a dwelling, and are therefore located in this new area, which does not exist within classical set theory.

Since fuzzy set theory is still set theory from a mathematical point of view, a modelling that starts from it will still not be sufficient to take into due account all these situations of doubt. On the other hand, since quantum cognition uses the mathematical formalism of quantum mechanics, it becomes possible to model this phenomenon of 'overextension of the probabilities'. We can make this clear without using the difficult and sophisticated mathematics of quantum mechanics, by directly considering the underlying structures of both classical and quantum theories, which is what we are now going to do.

\section{$5 \quad$ Classical and quantum}

We already noted that classical theories are based on set theory, so probabilities and measurements of typicalities will necessarily be mathematically expressed by what is called a 'measure on sets'. Such a measure, even in the most general case, behaves like the measure of a surface, if we can imagine these sets to be portions of a plane. We typically have the situation described in Figure 1, where two sets $A$ and $B$ are represented, with their intersection $A \cap B$ and union $A \cup B$. It is clear that the area of $A \cap B$ is always smaller or equal to the sum of the area of $A$ and the area of $B$ :

$$
\mu(A \cup B) \leq \mu(A)+\mu(B)
$$

This shows that the principle 'the whole $A \cup B$ is greater than the sum of its parts $A$ and $B$ ' never takes

place when the modelling is done from set theory. What we can also easily see from Figure 1 is that the area of $A \cap B$ is always lesser or equal to both the area of $A$ and the area of $B$ :

$$
\mu(A \cap B) \leq \mu(A) \quad \mu(A \cap B) \leq \mu(B)
$$


This shows that what happens with the 'conjunction effect', both in the case of the Linda problem and in the case of the pet-fish problem, never takes place when the modelling is done from set theory.

What is the underlying structure of quantum mathematics that does make it possible to model the 'conjunction phenomenon'? Well, 'state spaces' in quantum mechanics are described by 'Hilbert spaces', which are 'vector spaces over the complex numbers', possibly infinitely dimensional.

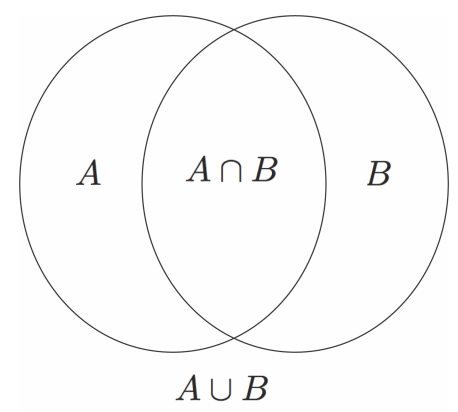

Figure 1: A graphical representation of two sets $A$ and $B$ in the plane, and of their intersection $A \cap B$ and union $A \cup B$. If $\mu$ is the 'Lebesgue measure', coinciding here with the standard measure of the area, we can read from the drawing that $\mu(A \cup B) \leq \mu(A)+\mu(B), \mu(A \cap B) \leq \mu(A)$ and $\mu(A \cap B) \leq \mu(B)$.

To be able to understand why the phenomenon of 'the whole that is larger than the sum of its parts' and of 'the conjunction that is larger than its elements', can be described by means of a quantum mathematical model, it will be enough to consider a simplified version of such model, and more precisely a two-dimensional real version that can be described on a plane. For this, let us consider four vectors lying on a same plane, which we call $A, B, C$ and $X$, as shown in Figure 2 .

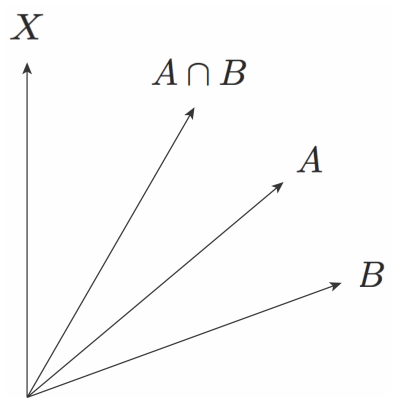

Figure 2: A graphical representation of four vectors $X, A \cap B, A$ and $B$, of a Hilbert space representing the situation of a quantum model able to model the conjunction phenomenon, i.e. the inequalities $\mu(A \cap B)>\mu(A)$ and $\mu(A \cap B)>\mu(B)$.

In the case of the Linda problem, $A$ represents 'bank teller', $B$ represents 'feminist', and $C$ represents 'bank teller and feminist', while $X$ represents 'Linda and her description'. For the pet-fish problem, $A$ represents Pet, $B$ represents Fish, and $C$ represents Pet-Fish, while $X$ represents Guppy.

Now, not only in quantum mechanics properties are represented by subspaces of the Hilbert space (which in our example are the one-dimensional subspaces generated by the different vectors on the plane), rather than by sets, but probabilities are also defined in a fundamentally different way. With the exception of the more elaborate quantum models developed following the 'hidden-measurement approach', where different 'measurement-interactions' are assumed to play a role in the selection of an outcome [13, 14, the quantum probabilities in standard quantum theory are not linked to a direct measure of properties or 
states, but to the angles that exist between subspaces of state vectors. It is not necessary here to specify the mathematical formula exactly used in our demonstration (the so-called Born rule), being enough to know, in our simple example on the plane, that if the angle between two vectors is $\theta=90^{\circ}$, which means that the two vectors are perpendicular to each other, then the probability associated with them is $P=0$. If the angle is $\theta=0^{\circ}$, i.e. if the vectors have the same direction, then the probability associated with them is equal to $P=1$. Also, when the angle decreases, from $\theta=90^{\circ}$ to $\theta=0^{\circ}$, the corresponding probability progressively increases, from $P=0$ to $P=1$, like the square of the cosine of the angle.

\section{Quantum modeling the Linda and pet-fish problems}

To be as specific as possible, let us assume that for the Linda problem the probability that Linda is a 'bank teller', a 'bank teller and feminist' and a 'feminist', were estimated by the interviewees to be $P_{\text {Linda }}(A)=$ $0.05, P_{\text {Linda }}(A \cap B)=0.4$ and $P_{\text {Linda }}(B)=0.7$, respectively. For the pet-fish problem, we assume that the degree of typicality of Guppy for Pet, Pet-Fish and Fish, were estimated to be $P_{P e t-F i s h}(A)=0.4$, $P_{\text {Pet-Fish }}(A \cap B)=0.9$ and $P_{P e t-F i s h}(B)=0.7$, respectively. In Figures 3 and 4 we have a depiction of the quantum models that can fit these data using a simple quantum vector space on the real plane.

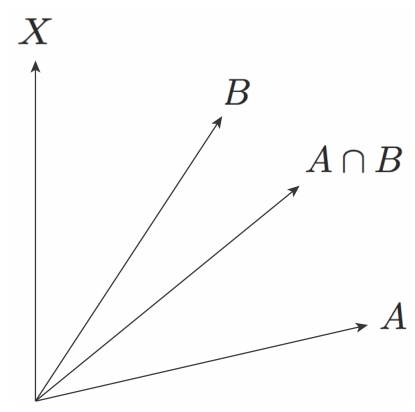

Figure 3: A graphical representation of four vectors $X, A \cap B, A$ and $B$, providing a quantum model for the data corresponding to the Linda, where $\theta(X, A)=33.21^{\circ}, \theta(X, A \cap B)=50.77^{\circ}$ and $\theta(X, B)=77.08^{\circ}$.

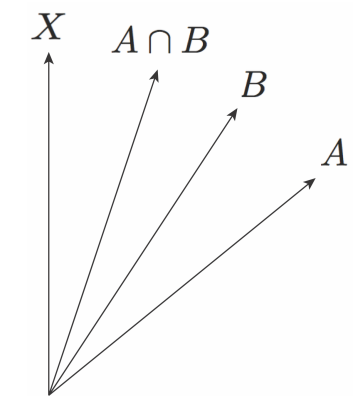

Figure 4: A graphical representation of four vectors $X, A \cap B, A$ and $B$, providing a quantum model for the data corresponding to the pet-fish problem, where $\theta(X, A \cap B)=18.43^{\circ}, \theta(X, B)=33.21^{\circ}$ and $\theta(X, A)=50.77^{\circ}$.

Indeed, in Figure 3 the angle between $X$ and $B, \theta(X, B)$, is the smallest, so corresponding with the highest probability $P_{\text {Linda }}(A)=0.7$, and using the quantum mechanical (Born) rule of probabilistic assignment we have:

$$
P(A)=\cos ^{2} \theta(X, A) \quad \Leftrightarrow \quad \theta(X, A)=\arccos \sqrt{P(A)} \quad\left(\bmod 180^{\circ}\right)
$$


so that we find the angles (modulo $\left.180^{\circ}\right): \theta(X, A)=33.21^{\circ}, \theta(X, A \cap B)=50.77^{\circ}$ and $\theta(X, A)=77.08^{\circ}$. Similarly, for the pet-fish problem, Figure 4 shows that the angle between $X$ and $A \cap B, \theta(X, A \cap B)$, is the smallest, so corresponding to the highest probability $P_{\text {Pet-Fish }}(A \cap B)=0.9$, so $(9)$ gives $\theta(X, A \cap B)=$ $18.43^{\circ}$, and we also obtain $\theta(X, B)=33.21^{\circ}$ and $\theta(X, A)=50.77^{\circ}$.

While it is true that more elaborated models are used in quantum cognition, with higher dimensional vector spaces and corresponding subspaces, it is already possible to see in this very simple two-dimensional example how the limitations that classical models carry with them (as a consequence of being formulated by means of set theory) can be easily overcome, using a probability calculus based on vectors spaces.

The conjunction fallacy, in the form identified by Tversky and Kahneman, was studied in quantum cognition by the group of Jerome Busemeyer at Indiana University [15, 16] and also as one of many other related phenomena of a non-classical nature that can occur in human decision-making processes, by the group of Emanuel Pothos at the City University of London, at the International Centre of Mathematical Modeling of Linnaeus University in Sweden (Andrei Khrennikov), and in the School of Business of Leicester University (Emanuel Haven) [16, 17, 18, 19, 20, 21]. The research into the way in which quantum models can describe concepts and their combinations, including the conjunction of concepts, was largely undertaken by the group at the Center Leo Apostel of VUB, in collaboration with the Universities of British Columbia, Leicester and Gdansk [12, 22, 23, 24, 25, 26, 27, 28, 29, 30, 31, 32, 33, 34. In particular, the research that led to the identification of the conjunction effect in texts of the World-Wide Web [31] has been important to find a way to identify and investigate the conjunction effect in visual perception, as I'm going to describe in the following section, and has also led to a quantum model for the World-Wide Web itself, which we called the QWeb [34].

\section{The conjunction effect in visual perception}

For the development of our quantum model for the World-Wide Web, we showed in [34] how a quantum-like 'entity of meaning' can be linked to a 'language entity consisting of printed documents', where we considered the latter as a collection of traces left behind by the former, in specific results of searches we described as measurements. In other words, we offered a perspective where a collection of documents such as the World-Wide Web is described as a space of manifestation of a more complex entity - the QWeb - that was the object of our modelling and for which we used inspiration and experience coming from previous studies on operational-realistic approaches to quantum mechanics and quantum modelling of human cognition and decision processes. We also emphasized in [34] that a consistent QWeb model must account for the observed correlations between words found in the printed documents, such as 'co-occurrences', since the latter are connected to 'meaning connections' that exist between the concepts associated with these words. In this respect, we showed that 'context and interference (quantum) effects' are necessary to explain the probabilities calculated by counting the relative numbers of documents containing specific words and the co-occurrence of these words [34].

It is from the insight we gained in the study of texts of the World-Wide Web that I can also propose here a quantitative modelling of the 'the meaning that is contained in entities of visual perception'. This general quantum modelling will also be applicable in the specific case where the visual entity is a photograph. Let us consider the specific photo of Figure 5 and use it to explain in some detail the idea behind the approach. Two striking 'conjunctions' can be seen in the photo that produce a 'conjunction effect'. The first is 'the bottle and the glass' and the second is 'the mother and the child'. Let us consider some elements that can make these conjunction effects clearer. Consider the concept of Thirst. If we consider a photo with only a bottle, or a glass, then we can suspect that Thirst will be evoked less effectively by looking at it than is the case for this photo, where both the glass and the bottle are present. For the conjunction of Mother and Child we can consider the element Love. A photo of a child alone or a mother alone will evoke much 
less the element of Love than the connection between mother and child shown in this photo.

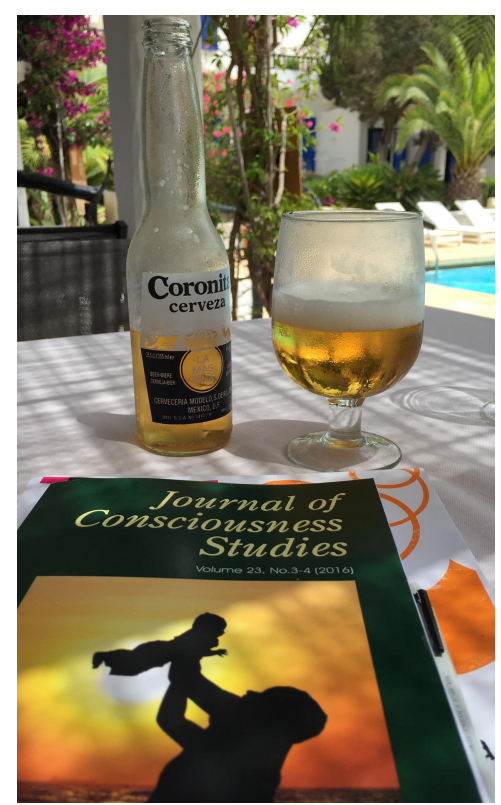

Figure 5: A photograph with different examples of conjunction effects (courtesy of Massimiliano Sassoli de Bianchi)

But there are also more subtle conjunction effects present in the picture. For example, the conjunction of Mind and Consciousness. There is the Spirit and Bottle and Consciousness as a modern name for Spirit, and the Journal of Consciousness Studies which is a new entity, whereas the beer in the bottle and glass is a very old alcoholic beverage. There is also a subtler connection between Mother and Child on the cover of the magazine: the depiction of mother and child explicitly shows a 'spatial connection', the mother has the child in her arms, and at the same time an emotional connection, the love between mother and child. There is also a connection between the bottle and the glass, on the one hand, spatially, by the action of someone who drinks, by standing on the same table, and also Bottle and Glass are emotionally/energetically connected, the bottle empties itself to fill the glass. Each of these elements also contains such conjunctions effects, that is, effects that indicate that 'the whole is greater than the sum of its parts'. By the analysis of this specific photograph it is easy to understand that every photo, and by extension each image, has built-in such conjunction effects, and as a consequence the 'whole that is greater than the sum of its parts' will be true for each image.

\section{The ontology of an image}

The insight that for each image the phenomenon of 'the whole that is greater than the sum of its parts' is present, will not create any surprise in the context of artistic interpretation of visual entities such as images. However, what we would like to put forward here is that from the perspective of quantum cognition and the associated mathematical models, it becomes possible to investigate this phenomenon quantitatively. Such an approach also allows us to understand in a more precise way what is going on in this respect with a visual entity, such as an image.

In the previous discussion, we used two specific ways to express what is going on that can bring us to the path of a deeper understanding of the conjunction effects in visual entities such as photos. In a first part of our discussion we used the expression "...will evoke much less the element of Love than the..." 
and later we wrote "...'contains' such conjunction effects...". These two verbs, 'evoking' and 'containing', are those that we would like to consider now in more detail. The first refers to a 'creative event' that is directly connected to the person looking at the photograph, or more generally, the person who interacts with the visual entity. It indicates that there is a creative action of the viewer. The second verb, 'contain', refers to the presence, 'independently of the creative action of the viewer', of an element of meaning in the photograph itself. Intuitively we know that both are present. It can be shown that quantum mechanics is a theory that describes both aspects in a way such that there is a balance between a 'creation aspect', introduced by the viewer, and a 'discovery aspect', which is already present and is independent of whether the photograph is looked at or not [13, 35, 36.

Physicists refer to 'the existing aspect' that is independent of the observer by means of the notion of 'state'. This corresponds to how we also use the notion of 'state' in our daily lives. For example, when we say that 'the car is in a worn state', we mean 'what the car is, regardless of what someone does with it'. Thus, 'state' refers to the 'ontology' of an entity. The creation aspect that an observer, a viewer when talking about a visual entity, adds to when interacting with an entity in a certain state, physicist will call it a 'measurement'. This is a notion that is used mainly in science, and one can feel the bias of science in it, where 'interacting with the ontological' corresponds to a measuring process. We will therefore not use the term 'measurement' and rather talk more generally about 'observing', 'perceiving' or 'interacting'. However, this does not mean that we cannot make use of the way quantum mechanics describes the act of 'measuring', to also describe our 'observing', 'perceiving' or 'interacting'. This is also what is done in quantum cognition when quantum mechanical models are developed to describe situations in human cognition. It is also in this way that we plan to use the quantum mechanical models to describe situations in visual perception, where the 'ontology' of the visual entity under consideration is accounted for by its 'state' and the observer's interaction by its 'measurement', as quantum mechanics does in the description of physical systems.

What is the ontology of a visual entity, and more specifically, 'what is the ontology of a photograph'? Thus, what is the meaning of a photograph that exists 'independently' of those who perceive or look at it? This is a question that we would like to examine in more detail now, because we can show how our previous analysis surprisingly provides an answer that would not have been obvious without it. First of all, the ontology of a photo is 'not' what is printed on the piece of paper that serves as a carrier for the photo, or is 'not' what appears as coloured pixels on a computer or TV screen. If that would be true, if the ontology of a photograph were to be equal to what is printed or appears on a screen, then 'the whole would not be greater than the sum of its parts', because what is printed, or what appears on a screen, is perfectly well described by set theory. It is enough to attribute the different points or pixels to the different sets and the situation presented in Figure 1 is then perfectly applicable.

The identification of conjunction effects gives us a hint of what the ontology of a visual entity is: it is the place the visual entity occupies in the global realm of human culture. Let me make this more concrete for the case of a photo. The ontology of a photograph, i.e. 'what a photograph is', regardless of whether it is viewed or not, is 'the place that the photograph occupies within the global realm of photos in human culture'. We can 'test' this definition of ontology of a photo. For this, let us consider the conjunction Mother and child of two images/concepts Mother and Child, and let us take 'Google Images' as a representation of the global realm of photos in human culture. If we type 'mother and child' into Google Images - more specifically the three words 'mother' 'and' and 'child', hence we did not force Google to search for the query of the exact combination, which would also have been an option -, we will find somewhat around 500 pictures showing a mother and a child, before others appear, where only a child or a mother are depicted separately. On the other hand, if we now connect two concepts that are not 'meaning-connected' in our human culture, by means of a conjunction, e.g. Giraffe and Salamander, and we type 'giraffe and salamander' in Google Images, we will find practically only photos on which only one 
of the two are depicted. This means that Giraffe and Salamander are very weakly meaning-connected in our human culture, whereas Mother and Child are very strongly meaning-connected. The conjunction effect thus refers to the 'meaning presence' of those ontological aspects of a photograph that are 'different from what has been printed or what is visible on a screen'. One can imagine that where the conjunction effects are present in a photograph, there is something that 'protrudes from the paper or emerges from the screen', inhabiting the higher-dimensional space of our global human culture. It is no coincidence that when we define the ontology of entities (in our case visual entities like photographs) in this way, quantum mechanics is able to model them. Indeed, the coherence that we observe in our human culture is very similar, in terms of substance, to the coherence characterizing the quantum entities and their interactions [43].

\section{Violation of the conjunction inequalities}

We can however identify, also in a real quantitative way, the presence of overextensions identical to those in the Linda and pet-fish problems, by making use of the Google Images search tool. We notice that for a search in Google Images usually around 5 pictures are shown in a row (for a typical screen size) and the number of rows is then around 80, which means that Google Images shows some 400 pictures. Let us first consider the conjunction effect connected to Mother, Child and Mother and Child. We make a Google Images search of the term 'mother' and keep the page of around 400 pictures open, then we make in another window of our browser a Google Images search of the term 'child', and keep also the page of around 400 pictures open, and finally in a third window of our browser we make a Google Images search of the exact sentence 'mother and child' and keep also in this case these around 400 pictures open.

As a sign of Love, we can look for the presence of an 'embrace' in the different pictures. I did this on September 21, 2017, and found the following results. The number $n$ (mother) of pictures open in the 'mother' search was 415 , and $n$ (embrace, mother) $=155$ showed an 'embrace', the number $n$ (child) of pictures open in the 'child' search was 475 , and $n$ (embrace, child) $=22$ showed an 'embrace', and the number $n$ (mother and child) of pictures open in the 'mother and child' search were 365 , and $n$ (embrace, mother and child $)=303$ showed an 'embrace'. If we consider the fractions of 'embraces' present as a good measure of the presence of Love, as a meaning, in the considered type of picture, we find

$$
\begin{aligned}
& P_{\text {Love }}(\text { Mother })=\frac{n(\text { embrace }, \text { mother })}{n(\text { mother })}=\frac{155}{415} \approx 0.37 \\
& P_{\text {Love }}(\text { Child })=\frac{n(\text { embrace }, \text { child })}{n(\text { child })}=\frac{22}{475} \approx 0.05 \\
& P_{\text {Love }}(\text { Mother and } \text { Child })=\frac{n(\text { embrace }, \text { mother and child })}{n(\text { mother and child })}=\frac{303}{365} \approx 0.83
\end{aligned}
$$

which shows that

$$
P_{\text {Love }}(\text { Mother and Child })>P_{\text {Love }}(\text { Mother }) \quad P_{\text {Love }}(\text { Mother and Child })>P_{\text {Love }}(\text { Child })
$$

for the notion Love, if we consider the presence of an 'embrace' as the sign for the notion of Love.

Let us consider now the concepts Bottle, Glass and Bottle and Glass, and as a sign for Thirst we verify whether some 'liquid' is present in the considered picture. We again use Google Images in three open browser windows for searches of 'bottle', 'glass' and 'bottle and glass', and count the number of pictures in each open browser window, and each time also the number of pictures where 'liquid' is present. This gives us a number of 325 pictures in the search window for 'bottle', with 52 showing the presence of 'liquid', a number of 390 pictures in the search window for 'glass', with 17 with showing the presence of 'liquid', 
and a number of 310 pictures in the search window for 'bottle and glass', with 92 showing the presence of 'liquid'. If we consider the fractions of those where some 'liquid' is present as a good measure of the meaning presence of Thirst in the considered type of pictures, we find

$$
\begin{aligned}
& P_{\text {Thirst }}(\text { Bottle })=\frac{n(\text { liquid }, \text { bottle })}{n(\text { bottle })}=\frac{52}{325} \approx 0.16 \\
& P_{\text {Thirst }}(\text { Glass })=\frac{n(\text { liquid }, \text { glass })}{n \text { (glass })}=\frac{17}{390} \approx 0.04 \\
& P_{\text {Thirst }}(\text { Bottle and Glass })=\frac{n(\text { liquid, bottle and glass })}{n(\text { bottle and glass })}=\frac{92}{310} \approx 0.30
\end{aligned}
$$

which shows that

$$
P_{\text {Thirst }}(\text { Bottle and Glass })>P_{\text {Thirst }}(\text { Bottle }) \quad P_{\text {Thirst }}(\text { Bottle and Glass })>P_{\text {Thirst }}(\text { Glass })
$$

for the notion of Thirst, if we consider the presence of 'liquid' as a valid sign for the presence of the notion Thirst.

So, we have proven the presence of 'emergence' for the concept of Love, for the combination of concepts Mother and Child, and we have done it also for the concept of Thirst, for the combination of concepts Bottle and Glass, in a quantitative way, by making use of the Google Images search tool on the World-Wide Web. More specifically, if Mother and Child appear together in an image this increases the probability that also an embrace as a sign of Love appears in that picture, as compared to such an embrace appearing as a sign of Love if pictures are considered containing Mother or containing Child. Hence it shows that combining Mother and Child within an image increases the probability of the emergence of Love in this image, and compared to such an emergence taking place in images containing Mother or images containing only Child. A similar phenomenon of 'increased emergence' for Thirst takes place when Bottle and Glass appear both in an image as compared to the emergence of Thirst for an image containing only Bottle or an image containing only Glass. This 'increased emergence', called 'overextension', can be modelled by the phenomenon of 'interference'. Indeed, the quantum theoretical models that were developed and shown to successfully account for this type of overextension in situations of cognitive science, such as the Linda problem and the pet-fish problem [12, 24, 29, 30, 37, 38, 39], which can equally be used in the present visual perception situation, precisely used interference effects as the main quantum effect to explain the deviation observed with respect to classical probabilities.

\section{Quantum entanglement in visual perception}

Encouraged by the results exposed in the foregoing sections, namely the violation of the conjunction inequalities leading to the identification of overextension in the probabilities calculated from data connected to images on the World-Wide Web, I was eager to explore more of the quantum nature of an image. The question that came to mind is "whether it were possible to identify the presence of quantum entanglement between different aspects of an image'. The violation of the conjunction inequalities made it possible to identify the presence of Linda and pet-fish problem type situations in visual perception. Could we use the same method of calculating probabilities using Google Images to violate a Bell type inequality and demonstrate the presence of quantum entanglement? This indeed turned out to be the case.

Making use of the example of the conceptual combination The Animal Acts, combining the two concepts Animal and Act, and different exemplars of this combination, then using Google Images, and the extraction of the corresponding probabilities as relative frequencies of appearances of the combinations of the exemplars, I was able to violate in a significant way the Clauser Horne Shimony Holt (CHSH) version of 
Bell's inequalities [40, in this way showing the presence of quantum entanglement in visual perception. In the following I will explain in detail how this violation comes about. But before that, it is worth observing that a violation of the CHSH inequality was shown to occur for the same combination of concepts The Animal Acts and the same set of exemplars than the one we will consider here, in cognitive experiments with human subjects [26]. The CHSH inequality is violated in case a specific term in it (se below) is smaller than -2 or bigger than 2, and the numerical violation obtained in [26] is equal to 2.4197, while as we are going to see I will obtain a numerical violation equal to 2.4107. That these violations are so numerically close, while the probabilities come about from completely different types of experiments, can be taken as a sign of the robustness of the presence of the entanglement, in the first case when the combined concepts are elements of the human language and in the second case when they are the visual elements of pictures.

I will explain now how this violation comes about. To calculate the term $E\left(A^{\prime}, B^{\prime}\right)+E\left(A, B^{\prime}\right)+$ $E\left(A^{\prime}, B\right)-E(A, B)$ that appears in the CHSH inequality

$$
-2 \leq E\left(A^{\prime}, B^{\prime}\right)+E\left(A, B^{\prime}\right)+E\left(A^{\prime}, B\right)-E(A, B) \leq 2
$$

we need to consider four experiments $e(A, B), e\left(A, B^{\prime}\right), e\left(A^{\prime}, B\right)$ and $e\left(A^{\prime}, B^{\prime}\right)$, each of them consisting in a coincidence experiment. For example, $e(A, B)$ is the experiment consisting in jointly performing the measurements of concepts $A$ and $B$. The quantities $E(A, B), E\left(A, B^{\prime}\right), E\left(A^{\prime}, B\right)$ and $E\left(A^{\prime}, B^{\prime}\right)$, which are averages, will then be calculated from the data gathered by the experiments $e(A, B), e\left(A, B^{\prime}\right), e\left(A^{\prime}, B\right)$ and $e\left(A^{\prime}, B^{\prime}\right)$, respectively, as it will be explained in the following.

I will first explain what the experiment $e(A, B)$ is. Consider for the concept Animal the two exemplars Horse and Bear, as outcomes, and for the concept Acts the two exemplars Growls and Whinnies, as outcomes. The four combinations The Horse Growls, The Horse Whinnies, The Bear Growls and The Bear Whinnies, where each of them is an exemplar of The Animal Acts, constitute the four outcomes of experiment $e(A, B)$, where $A$ and $B$ are jointly measured. To obtain the probabilities associated with these four outcomes, I proceeded as follows. In Google Images I made a search on 'horse growls', entering the two words one after the other in the Google bar, and saw that 400 images were shown to me by one hit of Google Images. I then carefully inspected each of the 400 images and counted the number of them on which I saw a 'horse that growls'. This of course contains some subjective appreciation of the images, because I only had access to visual information. Doing so, I concluded that of the 400 images there were 4 possibly showing 'a horse that is growling' (instead of whinnying, which is of course the most common noise for a horse to make, or instead of the horse being shown making no sound at all, or instead of the image showing no horse at all).

I then used the same method as in the foregoing sections to come to a probability, namely we divide the number of images showing a horse that growls by the total number of images inspected. This gives $P\left(A_{1}, B_{1}\right)=\frac{4}{400}=0.01$. The second sub experiment consists of making a search on Google Images for 'horse whinnies'. This time 391 images showed up as search results. A careful verification of each image gave the count of 39 of them showing 'a horse that whinnies'. Hence, the probability that I was able to derive for this sub experiment is $P\left(A_{1}, B_{2}\right)=\frac{39}{391} \approx 0.0997$. Let me mention that I use $A_{1}$ to indicate the exemplar Horse for Animal and $A_{2}$ to indicate the exemplar Bear for Animal. Analogously $B_{1}$ indicates Growls for Acts and $B_{2}$ indicates Whinnies for Acts. The third sub experiment of $e(A, B)$ consists of making a search on Google Images for 'bear growls'. This time 380 images appeared after the search. I could count 142 of them showing 'a bear that growls'. Hence this led me to $P\left(A_{2}, B_{1}\right)=\frac{142}{380} \approx 0.3737$. The fourth sub experiment of $e(A, B)$ consists of making a search on Google Images for 'bear whinnies', and 389 images appeared after the search. I counted 2 of them able to be interpreted as 'a bear that whinnies'. Hence this lead me to $P\left(A_{2}, B_{2}\right)=\frac{2}{389} \approx 0.0050$.

A comment is at place now to specify the way these counts have been done. A first remark is that they turn out to be rather objective, what would perhaps not be expected at first sight. Indeed, I asked 
several friends to also look over the pictures and give their estimations. It turned out that a rather good agreement on the numbers of pictures where one imagines 'the horse to be growling', or 'the horse to be whinnying' or 'the bear to be growling', or 'the bear to be whinnying' was obtained. And this was also the case for all the other appearances of one of the considered exemplars of The Animal Acts in the experiments we will consider in the following. What is however more important to mention is the following. That a picture contained or not 'a horse whinnying' or 'a bear growling' is really quite objective, because these are two common acts of a horse and a bear. It is more difficult to make a decision whether a specific image is about the less common happenings of 'the horse growling' or 'the bear whinnying'. With respect to these non common situations, and they appear for each of the sub experiments, we decided to count an image as positive for it, whenever it was possible to imagine the not so common acts of said animals as pictured in the images. Even such an easygoing attitude with respect to the uncommon exemplars led to small numbers of their appearances, because it was very easy in each case to definitely decide that the uncommon exemplar was 'not' pictured. That this is a valuable way to proceed stems from the following fact. Each time such an image is counted and the interpretation would be too wide, e.g. one imagines that the horse on the image could be growling while if one would have been present when the picture was taken one would have heard this not to be the case, this will only affect the term in the CHSH inequality in a way that the latter will be 'less easily violated'. In other words, errors on measurements for the less common exemplars, due to a too broad interpretation, always push the term in the CHSH inequality towards the direction of a value closer to be contained in the interval $[-2,+2]$ where no violation occurs.

This aspect of the CHSH inequality, its enormous robustness for errors, is also the one explaining why the violation measured with coupled spins in quantum theory was such an important happening, indicating the presence of quantum entanglement in the physical micro-world [42. Indeed, also for spins it was true that errors due to imperfections of the experiments would only push the CHSH term in the inequality further away from its violation. I made screen pictures of all of the images indicating the ones that were withheld for 'horse growling', and also screen pictures of all the other Google Images search results and how I came to the decision to count the ones that confirm the specific exemplar of The Animal Acts. Hence, any reader can receive these images on request to verify how the decisions to withhold images was made.

For this first sub experiment $e(A, B)$ we get four probabilities $\mathcal{P}\left(A_{1}, B_{1}\right), \mathcal{P}\left(A_{1}, B_{2}\right), \mathcal{P}\left(A_{2}, B_{1}\right)$ and $\mathcal{P}\left(A_{2}, B_{2}\right)$, respectively connected to the chance to find a 'horse that growls', a 'horse that whinnies', a 'bear that growls' and a 'bear that whinnies', in the collection of Google Images obtained when the searches are made as explained above. However, to formulate the $\mathrm{CHSH}$ inequality we need to further normalize these quantities. More specifically, we need estimations of "what is the probability that one of the four will appear if we know that at least one of them is appearing'. We can obtain these renormalised probabilities by dividing each of the now calculated probability $\mathcal{P}$ by their sum (note that all decimal numbers indicated below are approximate numbers, so the equality symbols should be more precisely replaced by approximate equality symbols):

$$
\mathcal{S}(A, B)=\mathcal{P}\left(A_{1}, B_{1}\right)+\mathcal{P}\left(A_{1}, B_{2}\right)+\mathcal{P}\left(A_{2}, B_{1}\right)+\mathcal{P}\left(A_{2}, B_{2}\right)=0.4884
$$

This gives:

$$
\begin{array}{ll}
P\left(A_{1}, B_{1}\right)=\frac{\mathcal{P}\left(A_{1}, B_{1}\right)}{\mathcal{S}(A, B)}=0.0205 & P\left(A_{1}, B_{2}\right)=\frac{\mathcal{P}\left(A_{1}, B_{2}\right)}{\mathcal{S}(A, B)}=0.2042 \\
P\left(A_{2}, B_{1}\right)=\frac{\mathcal{P}\left(A_{2}, B_{1}\right)}{\mathcal{S}(A, B)}=0.7651 & P\left(A_{2}, B_{2}\right)=\frac{\mathcal{P}\left(A_{2}, B_{2}\right)}{\mathcal{S}(A, B)}=0.0103
\end{array}
$$

The obtained expectation value $E(A, B)$ is then

$$
E(A, B)=P\left(A_{1}, B_{1}\right)-P\left(A_{1}, B_{2}\right)-P\left(A_{2}, B_{1}\right)+P\left(A_{2}, B_{1}\right)=-0.9385
$$


The idea is that the choice for Animal which is Horse is given the value 1, while the choice for Animal which is Bear is given the value -1. Similarly, the choice for Acts which is Growls is given the value 1, and the choice for Acts which is Whinnies is given the value -1. Then, combining these values, we obtain that the choice The Horse Growls is associated with the value 1, obtained by multiplying the value 1 for Horse with the value 1 for Growls. Similarly, the choice The Horse Whinnies is -1 (multiplying 1 with -1), the choice The Bear Growls is -1 (multiplying -1 with 1), and the choice The Bear Whinnies is 1 (multiplying -1 with -1$)$. Then, $E(A, B)$ is the 'expected value' given by the probabilities $P\left(A_{1}, B_{1}\right)$, $P\left(A_{1}, B_{2}\right), P\left(A_{2}, B_{1}\right)$ and $P\left(A_{2}, B_{1}\right)$ of each of these values. Hence $E(A, B)=-0.9385$ means that there is a strong anti-correlation, and indeed, Horse anti-correlates with Growls and Bear anti-correlates with Whinnies.

To define the three remaining experiments $e\left(A, B^{\prime}\right), e\left(A^{\prime}, B\right)$ and $e\left(A^{\prime}, B^{\prime}\right)$ that are needed to calculate the CHSH inequality term, we consider two different exemplars for Animal as well as for Acts, namely Tiger and Cat and Snorts and Meows. The three experiments are now defined as follows, $e\left(A, B^{\prime}\right)$ is the experiment where the old exemplars for Animal, Horse and Bear, are combined with the new exemplars for Acts, Snorts and Meows, $e\left(A^{\prime}, B\right)$ is the experiment where the new exemplars for Animal, Tiger and Cat, are combined with the old exemplars for Acts, Growls and Whinnies, and $e\left(A^{\prime}, B^{\prime}\right)$ is the experiments where for both Animal and Acts the new exemplars are combined, hence Tiger and Cat with Snorts and Meows.

So, for the experiment $e\left(A, B^{\prime}\right)$, I did Google Images searches for 'horse snorts', delivering 407 images of which 41 show a snorting horse; for 'horse meows', delivering 403 images of which 4 show a meowing horse; for 'bear snorts', delivering 385 images of which 23 show a snorting bear; and for 'bear meows', delivering 405 images of which 5 show a meowing bear. This gives $\mathcal{P}\left(A_{1}, B_{1}^{\prime}\right)=0.1007, \mathcal{P}\left(A_{1}, B_{2}^{\prime}\right)=0.0099$, $\mathcal{P}\left(A_{2}, B_{1}^{\prime}\right)=0.0597$ and $\mathcal{P}\left(A_{2}, B_{2}^{\prime}\right)=0.0123$, and hence

$$
\begin{aligned}
& \mathcal{S}\left(A, B^{\prime}\right)=\mathcal{P}\left(A_{1}, B_{1}^{\prime}\right)+\mathcal{P}\left(A_{1}, B_{2}^{\prime}\right)+\mathcal{P}\left(A_{2}, B_{1}^{\prime}\right)+\mathcal{P}\left(A_{2}, B_{2}^{\prime}\right)=0.1827 \\
& P\left(A_{1}, B_{1}^{\prime}\right)=\frac{\mathcal{P}\left(A_{1}, B_{1}^{\prime}\right)}{\mathcal{S}\left(A, B^{\prime}\right)}=0.5512 \quad P\left(A_{1}, B_{2}^{\prime}\right)=\frac{\mathcal{P}\left(A_{1}, B_{2}^{\prime}\right)}{\mathcal{S}\left(A, B^{\prime}\right)}=0.0543 \\
& P\left(A_{2}, B_{1}^{\prime}\right)=\frac{\mathcal{P}\left(A_{2}, B_{1}^{\prime}\right)}{\mathcal{S}\left(A, B^{\prime}\right)}=0.3269 \quad P\left(A_{2}, B_{2}^{\prime}\right)=\frac{\mathcal{P}\left(A_{2}, B_{2}^{\prime}\right)}{\mathcal{S}\left(A, B^{\prime}\right)}=0.0676 \\
& E\left(A, B^{\prime}\right)=P\left(A_{1}, B_{1}^{\prime}\right)-P\left(A_{1}, B_{2}^{\prime}\right)-P\left(A_{2}, B_{1}^{\prime}\right)+P\left(A_{2}, B_{1}^{\prime}\right)=0.2376
\end{aligned}
$$

For the experiment $e\left(A^{\prime}, B\right)$, I did Google Images searches for 'tiger growls', delivering 399 images of which 219 show a growling tiger; for 'tiger whinnies', delivering 402 images of which 3 show a whinnying tiger; for 'cat growls', delivering 405 images of which 78 show a growling cat; and for 'cat whinnies', delivering 402 images of which 1 shows a whinnying cat. This gives $\mathcal{P}\left(A_{1}^{\prime}, B_{1}\right)=0.5489, \mathcal{P}\left(A_{1}^{\prime}, B_{2}\right)=0.0075$, $\mathcal{P}\left(A_{2}^{\prime}, B_{1}\right)=0.1926$ and $\mathcal{P}\left(A_{2}^{\prime}, B_{2}\right)=0.0025$, and hence

$$
\begin{aligned}
& \mathcal{S}\left(A^{\prime}, B\right)=\mathcal{P}\left(A_{1}^{\prime}, B_{1}\right)+\mathcal{P}\left(A_{1}^{\prime}, B_{2}\right)+\mathcal{P}\left(A_{2}^{\prime}, B_{1}\right)+\mathcal{P}\left(A_{2}^{\prime}, B_{2}\right)=0.7514 \\
& P\left(A_{1}^{\prime}, B_{1}\right)=\frac{\mathcal{P}\left(A_{1}^{\prime}, B_{1}\right)}{\mathcal{S}\left(A^{\prime}, B\right)}=0.7305 \quad P\left(A_{1}^{\prime}, B_{2}\right)=\frac{\mathcal{P}\left(A_{1}^{\prime}, B_{2}\right)}{\mathcal{S}\left(A^{\prime}, B\right)}=0.0099 \\
& P\left(A_{2}^{\prime}, B_{1}\right)=\frac{\mathcal{P}\left(A_{2}^{\prime}, B_{1}\right)}{\mathcal{S}\left(A^{\prime}, B\right)}=0.2563 \quad P\left(A_{2}^{\prime}, B_{2}\right)=\frac{\mathcal{P}\left(A_{2}^{\prime}, B_{2}\right)}{\mathcal{S}\left(A^{\prime}, B\right)}=0.0033 \\
& E\left(A^{\prime}, B\right)=P\left(A_{1}^{\prime}, B_{1}\right)-P\left(A_{1}^{\prime}, B_{2}\right)-P\left(A_{2}^{\prime}, B_{1}\right)+P\left(A_{2}^{\prime}, B_{1}\right)=0.4675
\end{aligned}
$$

Finally, for the experiment $e\left(A^{\prime}, B^{\prime}\right)$, I did Google Images searches for 'tiger snorts', delivering 400 images of which 30 show a snorting tiger; for 'tiger meows', delivering 403 images of which 10 show a meowing tiger; for 'cat snorts', delivering 392 images of which 15 show a snorting cat; and for 'cat meows', delivering 399 images 
of which 161 show a meowing cat. This gives $\mathcal{P}\left(A_{1}^{\prime}, B_{1}^{\prime}\right)=0.075, \mathcal{P}\left(A_{1}^{\prime}, B_{2}^{\prime}\right)=0.0248, \mathcal{P}\left(A_{2}^{\prime}, B_{1}^{\prime}\right)=0.0383$ and $\mathcal{P}\left(A_{2}^{\prime}, B_{2}^{\prime}\right)=0.4035$, and hence

$$
\begin{aligned}
& \mathcal{S}\left(A^{\prime}, B^{\prime}\right)=\mathcal{P}\left(A_{1}^{\prime}, B_{1}^{\prime}\right)+\mathcal{P}\left(A_{1}^{\prime}, B_{2}^{\prime}\right)+\mathcal{P}\left(A_{2}^{\prime}, B_{1}^{\prime}\right)+\mathcal{P}\left(A_{2}^{\prime}, B_{2}^{\prime}\right)=0.5416 \\
& P\left(A_{1}^{\prime}, B_{1}^{\prime}\right)=\frac{\mathcal{P}\left(A_{1}^{\prime}, B_{1}^{\prime}\right)}{\mathcal{S}\left(A^{\prime}, B^{\prime}\right)}=0.1385 \quad P\left(A_{1}^{\prime}, B_{2}^{\prime}\right)=\frac{\mathcal{P}\left(A_{1}^{\prime}, B_{2}^{\prime}\right)}{\mathcal{S}\left(A^{\prime}, B^{\prime}\right)}=0.0458 \\
& P\left(A_{2}^{\prime}, B_{1}^{\prime}\right)=\frac{\mathcal{P}\left(A_{2}^{\prime}, B_{1}^{\prime}\right)}{\mathcal{S}\left(A^{\prime}, B^{\prime}\right)}=0.0707 \quad P\left(A_{2}^{\prime}, B_{2}^{\prime}\right)=\frac{\mathcal{P}\left(A_{2}^{\prime}, B_{2}^{\prime}\right)}{\mathcal{S}\left(A^{\prime}, B^{\prime}\right)}=0.7450 \\
& E\left(A^{\prime}, B^{\prime}\right)=P\left(A_{1}^{\prime}, B_{1}^{\prime}\right)-P\left(A_{1}^{\prime}, B_{2}^{\prime}\right)-P\left(A_{2}^{\prime}, B_{1}^{\prime}\right)+P\left(A_{2}^{\prime}, B_{1}^{\prime}\right)=0.7671
\end{aligned}
$$

We have now all available measurements and their corresponding calculations of probabilities and expectation values to calculate the term of the CHSH inequality, and this gives

$$
E\left(A^{\prime}, B^{\prime}\right)+E\left(A, B^{\prime}\right)+E\left(A^{\prime}, B\right)-E(A, B)=2.4107
$$

Hence, the CHSH inequality (18) is manifestly violated.

\section{Entanglement and meaning connection}

The violation of the conjunction inequalities indicates the presence of 'emergent meaning'. Linda fits this emergent meaning well for the conjunction 'bank teller' and 'active on the feminist movement' and that is why systematically the so-called conjunction fallacy is made when the corresponding experiment is done. Guppy fits the emergent meaning well for the conjunction of 'pet' and 'fish', and tomato resonates with the emergent meaning of 'fruits' and 'vegetables'. We can also understand the violation of Bell's inequalities and hence the identification of the presence of entanglement by reflecting about the meaning content of the considered situation. Let me illustrate this also by analysing some of the images that showed up when I performed the Google Images searches described in detail in the foregoing section and leading to the probabilities violating the CHSH version of Bell's inequalities.

In a search for 'horse whinnies' the typical image that showed up was like the one illustrated in Figure6, where one can see a horse whinny with its mouth being shaped in the typical form.

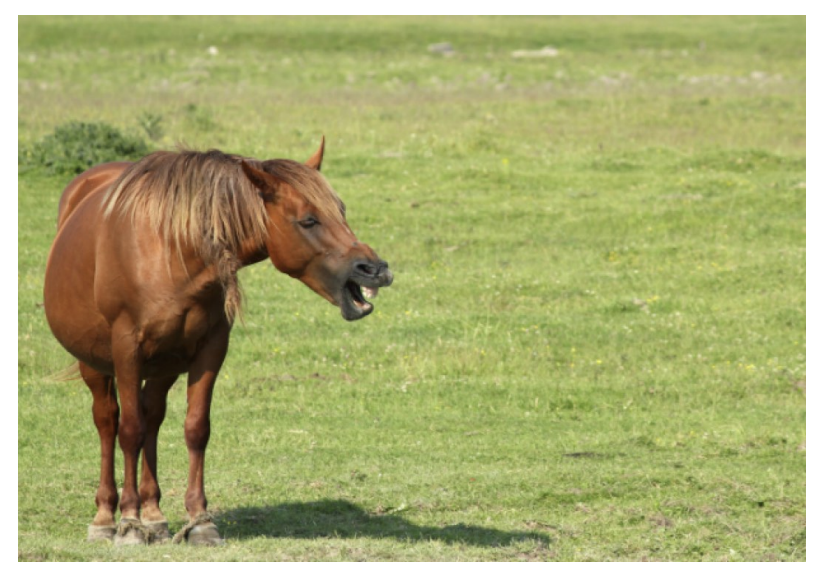

Figure 6: A typical image showing up when a search for 'horse whinnies' is being made in Google Images

Also for 'bear growls', 'tiger growls' and 'cat meows' the typical images showing these behaviors will appear, and the reader is invited to try this out in Google Images. Certainly, 'bear growls' gives rise to an impressive 
amount of pictures of heavily growling bears. However, even for a search of 'cat meows' also images will always appear in the search that do not show a 'meowing cat'. For example, the image in Figure 7 is the 19th image after 18 meowing cats.

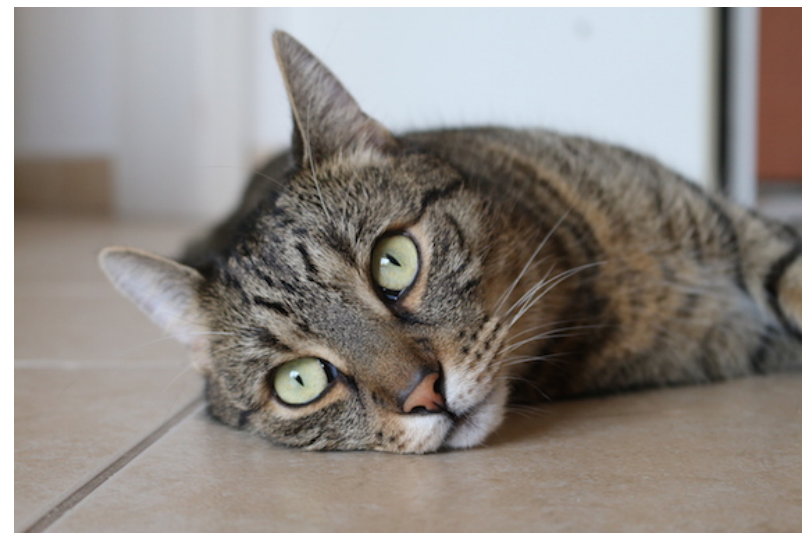

Figure 7: The 19th image appearing in a search of 'cat meows' in Google Images is no longer a cat that meows.

In the first experiment $e(A, B)$ these two exemplars, 'the horse whinnies' and 'the bear growls', are the dominant ones in terms of appearance, while the other two exemplars, 'the horse growls' and 'the bear whinnies', are very uncommon, and we have counted some of them mostly because some images might lend themselves to be interpreted in this way. It is also this rather strong variation of dominance of two of the exemplars versus the uncommon appearance of two of the other exemplars that brings the expectation value $E(A, B)$ to be close to -1 , which is an important factor for the CHSH inequalities to be able to be violated. It is of course the 'meaning connection' between Animal and Acts which is at the origin of this phenomenon of dominance of two of the exemplars and the uncommon appearance of the other two. However, connection in meaning is not enough to violate the CHSH inequalities, we can see this easily by considering a special case, where we take $A^{\prime}=A$ and $B^{\prime}=B$. Since then $E\left(A^{\prime}, B^{\prime}\right)=E\left(A, B^{\prime}\right)=E\left(A^{\prime}, B\right)=E(A, B)$ the term in the CHSH inequality becomes equal to $2 E(A, B)$, and since $-1 \leq E(A, B) \leq+1$, the inequality will never be violated.

This special case shows that to violate the inequalities, a meaning connection is necessary, but the source of it, in our case the conceptual combination The Animal Acts, needs also to be able to respond to different experiments in different ways. This 'creation aspect' of being ble to 'respond to different experiments in different ways' is also very typical of the potentiality contained in a quantum state [43, 44, 45]. This potentiality is carried by the very conceptual nature of abstract entities such as Animal and Acts: they can realise the more concrete states of Horse, Bear, Tiger and Cat for the case of Animal, and Growls, Whinnies, Snorts and Meows for the case of Acts, each time in a different way (as in a process of symmetry breaking), being only guided by the existing meaning connection.

\section{Conclusion}

When I worked on the experiments about the after image, during my Master studies in visual arts [47, I would not have imagined that soon after I would be studying the violation of conjunction and Bell's inequalities due to the presence of quantum superposition and quantum entanglement in visual perception. But I know now that additionally to my quest for the heart of an image, guided by my long lasting fascination for the content and meaning carried by an image, a long-running circle was coming to its 
closure. I remember how my father would ask me and my friends, playing as children in the garden not far away from where he was working, how we would think about an 'tree house' whether we believed it to be rather a 'building' or a 'dwelling' or somewhat both. I also remember him asking us about 'mint' and whether we considered it to be a plant or food or rather both. He also told us about the 'pet-fish', and how 'guppy' and 'goldfish' behaved weirdly with respect to it, and how he was investigating whether this behaviour could be understood by making use of quantum theory. I remember how these intermezzi in our playing would also lead to listening to a presentation of the double-slit experiment and the weird behaviour of quantum particles. However, I would never have fathomed then that my studies in visual art and my fascination for the ontology of an image would come to be connected in such a meaningful way to what my father was presenting to me and my friends in those days. But so it was.

\section{References}

[1] Aerts Argüelles J. (2017). Gestalten van Emergentie in Visuele Perceptie. Een Zoektocht in Kunst en Wetenschap. Master Thesis. KASK, Ghent, Belgium.

[2] Sassoli de Bianchi, M. (2011). Ephemeral properties and the illusion of microscopic particles. Foundations of Science, 16, pp. 393-409.

[3] Heisenberg, W. (1969). Der Teil und das Ganze: Gespräche im Umkreis der Atomphysik. Munchen: Piper Verlag GmbH.

[4] Einstein A., Podolsky B., Rosen N. (1935). Can quantum-mechanical description of physical reality be considered complete?. Physical Review, 47, pp. 777-780.

[5] Bell J. S. (1987). Speakable and Unspeakable in Quantum Mechanics. Cambridge, UK: Cambridge University Press.

[6] Feynman, R. (1965). The Character of Physical Law. New York: Modern Library. See Chapter 6, Probability and Uncertainty - The Quantum Mechanical View of Nature, p. 129.

[7] Tversky, A. and Kahneman, D. (1982). Judgments of and by representativeness. In D. Kahneman, P. Slovic \& A. Tversky (Eds.), Judgment under Uncertainty: Heuristics and Biases. Cambridge, UK: Cambridge University Press.

[8] Moro, R. (2009). On the nature of the conjunction fallacy. Synthese, 171, pp. 1-24.

[9] Osherson, D. N. and Smith, E. E. (1981). On the adequacy of prototype theory as a theory of concepts. Cognition, 9, pp. 35-58.

[10] Sozzo, S. (2015). Conjunction and negation of natural concepts: A quantum-theoretic modeling. Journal of Mathematical Psychology, 66, pp. 83-102.

[11] Hampton, J. A. (1988). Overextension of conjunctive concepts: Evidence for a unitary model for concept typicality and class inclusion. Journal of Experimental Psychology: Learning, Memory, and Cognition, 14, pp. 12-32.

[12] Aerts, D. (2009). Quantum structure in cognition. Journal of Mathematical Psychology, 53, pp. 314348.

[13] Aerts, D. and Sassoli de Bianchi, M. (2015). The unreasonable success of quantum probability I: Quantum measurements as uniform fluctuations. Journal Mathematical Psychology, 67, pp. 51-75.

[14] Aerts, D. and Sassoli de Bianchi, M. (2015). The unreasonable success of quantum probability II: Quantum measurements as universal measurements Journal Mathematical Psychology, 67, pp. 76-90.

[15] Busemeyer, J. R., Pothos, E. M., Franco, R. and Trueblood. J. S. (2011). A quantum theoretical explanation for probability judgment errors. Psychological Review, 118, pp. 193-218.

[16] Trueblood, J. S. and Busemeyer, J. R. (2011). A quantum probability account of order effects in inference. Cognitive Science, 35, pp. 1518-1552. 
[17] Busemeyer, J. and Bruza, P. (2012). Quantum Models of Cognition and Decision. Cambridge, UK: Cambridge University Press.

[18] Pothos, E. M. and Busemeyer, J. R. (2009). A quantum probability explanation for violations of 'rational' decision theory. Proceedings of the Royal Society B: Biological Sciences, 276, pp. 2171-2178.

[19] Pothos, E. M. and Busemeyer, J. R. (2013). Can quantum probability provide a new direction for cognitive modeling. Behavioral and Brain Sciences, 36, pp. 255-274.

[20] Khrennikov, A. (2010). Ubiquitous Quantum Structure: From Psychology to Finances. Berlin: Springer.

[21] Haven, E. and Khrennikov, A. (2013). Quantum Social Science. Cambridge, UK: Cambridge University Press.

[22] Aerts, D. and Gabora, L. (2005). A theory of concepts and their combinations I. The structure of the sets of contexts and properties. Kybernetes, 34, pp. 192-221.

[23] Aerts, D. and Gabora, L. (2005). A theory of concepts and their combinations II. A Hilbert Space representation. Kybernetes, 34, pp. 167-191.

[24] Aerts, D., Gabora, L. and Sozzo, S. Concepts and their dynamics: A quantum-theoretic modeling of human thought. Topics in Cognitive Science, 5, pp. 737-772.

[25] Aerts, D. and Czachor, M. (2004). Quantum aspects of semantic analysis and symbolic artificial intelligence. Journal of Physics A: Mathematical and Theoretical, 37, pp. L123-L132.

[26] Aerts, D. and Sozzo, S. (2011). Quantum structure in cognition: Why and how concepts are entangled. Quantum Interaction, Lecture Notes in Computer Science, 7052, pp. 116-127.

[27] Aerts, D., Broekaert, J. and Gabora, L. (2011). A case for applying an abstracted quantum formalism to cognition. New Ideas in Psychology, 29, pp. 136-146.

[28] Gabora, L. and Kitto, K. (2016). Towards a quantum theory of humour. Frontiers in Physics, 4. doi:10.3389/fphy.2016.00053.

[29] Sozzo, S. (2014). A quantum probability explanation in Fock space for borderline contradictions. Journal of Mathematical Psychology, 58, pp. 1-12.

[30] Aerts, D., Aerts Arguëlles, J., Beltran, L., Distrito, I., Sassoli de Bianchi, M., Sozzo, S., and Veloz, T. (2017). Context and interference effects in the combinations of natural concepts. CONTEXT 201\%: Modeling and Using Context. Lecture Notes in Computer Science, 10257, pp. 677-690. doi:10.1007/ 978-3-319-57837-8_54

[31] Aerts, D., Aerts Arguëlles, J., Beltran, L., Beltran, L., Sassoli de Bianchi, M., Sozzo, S., and Veloz, T. (2017). Testing quantum models of conjunction fallacy on the World-Wide Web. International Journal of Theoretical Physics, first online. doi:10.1007/s10773-017-3288-8.

[32] Aerts, D., Aerts Arguëlles, J., Beltran, L., Geriente, S., Sassoli de Bianchi, M., Sozzo, S., and Veloz, T. (2017). Spin and wind directions I: Identifying entanglement in nature and cognition. Foundations of Science, first online. doi:10.1007/s10699-017-9528-9.

[33] Aerts, D., Aerts Arguëlles, J., Beltran, L., Geriente, S., Sassoli de Bianchi, M., Sozzo, S., and Veloz, T. (2017). Spin and wind directions II: A Bell state quantum model. Foundations of Science, first online. doi:10.1007/s10699-017-9530-2.

[34] Aerts, D., Aerts Arguëlles, J., Beltran, L., Beltran, L., Distrito, I., Sassoli de Bianchi, M., Sozzo, S., and Veloz, T. (2017). Towards a quantum World-Wide Web. Submitted to Theoretical Computer Science. Preprint at arXiv:1703.06642 [cs.AI].

[35] Aerts, D. (1998). The entity and modern physics: the creation-discovery view of reality. In E. Castellani (Ed.), Interpreting Bodies: Classical and Quantum Objects in Modern Physics (pp. 223-257). Princeton: Princeton University Press. 
[36] Aerts, D. and Coecke, B. (1999). The creation-discovery-view: towards a possible explanation of quantum reality. In M. L. Dalla Chiara, R. Giuntini and F. Laudisa (Eds.), Language, Quantum, Music: Selected Contributed Papers of the Tenth International Congress of Logic, Methodology and Philosophy of Science, Florence, August 1995. Dordrecht: Springer.

[37] Aerts, D., Sozzo, S. and Veloz, T. (2015a). Quantum structure in cognition and the foundations of human reasoning. International Journal of Theoretical Physics, 54, pp. 4557-4569.

[38] Aerts, D., Sozzo, S. and Veloz, T. (2015b). Quantum structure of negation and conjunction in human thought. Frontiers in Psychology, 6, 1447.

[39] Aerts, D., Sozzo, S. and Veloz, T. (2015c). A new fundamental evidence of non-classical structure in the combination of natural concepts. Philosophical Trasactions of the Royal Society A, 374, 20150095.

[40] Clauser, J. F., Horne, M. A., Shimony, A. \& Holt, R.A. (1969). Proposed experiment to test local hidden-variable theories. Physical Review Letters 23, pp. 880-884.

[41] Aerts, D. and Sozzo, S. (2014). Quantum entanglement in concept combinations. International Journal of Theoretical Physics, 53, pp. 3587-3603.

[42] Aspect, A., Grangier, P. and Roger, G. (1981). Experimental tests of realistic local theories via Bell's theorem. Physical Review Letters, 47, pp. 460-463.

[43] Aerts, D. (2009b). Quantum particles as conceptual entities: A possible explanatory framework for quantum theory. Foundations of Science, 14, pp. 361-411.

[44] Aerts, D. (2010a). Interpreting quantum particles as conceptual entities. International Journal of Theoretical Physics 49, pp. 2950-2970.

[45] Aerts, D. (2010b). A potentiality and conceptuality interpretation of quantum physics. Philosophica 83 , pp. $15-52$.

[46] Aerts, D. (2013). La mecánica cuántica y la conceptualidad: Sobre materia, historias, semántica y espacio-tiempo. Scientiae Studia 11, pp. 75-100. Translated from: Aerts, D. (2011). Quantum theory and conceptuality: Matter, stories, semantics and space-time. arXiv:1110.4766 [quant-ph], October 2011.

[47] Aerts Argüelles J. (2017). Eigengrau. http://www.jonitoaertsarguelles.com. End Work Master Thesis. KASK, Ghent, Belgium. 